\title{
Approximation with Kronecker Products
}

\author{
Charles Van Loan and Nikos Pitsianis \\ Department of Computer Science \\ Cornell University \\ Ithaca, New York 14853
}

\begin{abstract}
Let $A$ be an $m$-by- $n$ matrix with $m=m_{1} m_{2}$ and $n=n_{1} n_{2}$. We consider the problem of finding $B \in \mathbb{R}^{m_{1} \times n_{1}}$ and $C \in \mathbb{R}^{m_{2} \times n_{2}}$ so that $\|A-B \otimes C\|_{F}$ is minimized. This problem can be solved by computing the largest singular value and associated singular vectors of a permuted version of $A$. If $A$ is symmetric, definite, non-negative, or banded, then the minimizing $B$ and $C$ are similarly structured. The idea of using Kronecker product preconditioners is briefly discussed.
\end{abstract}

\section{Introduction}

Suppose $A \in \mathbb{R}^{m \times n}$ with $m=m_{1} m_{2}$ and $n=n_{1} n_{2}$. This paper is about the minimization of

$$
\phi_{A}(B, C)=\|A-B \otimes C\|_{F}^{2}
$$

where $B \in \mathbb{R}^{m_{1} \times n_{1}}, C \in \mathbb{R}^{m_{2} \times n_{2}}$, and " $\otimes$ " denotes the Kronecker product.

Our interest in this problem stems from preliminary experience with Kronecker product preconditioners in the conjugate gradient setting. Suppose $A \in \mathbb{R}^{n \times n}$ with $n=n_{1} n_{2}$ and that $M$ is the preconditioner. For this solution process to be successful, the preconditioner should "capture" the essence of $A$ as much as possible subject to the constraint that a linear system $M z=r$ is "easy" to solve. In our context, we capture $A$ through the minimization $\phi_{A}(B, C)$ with $B \in \mathbb{R}^{n_{1} \times n_{1}}$ and $C \in \mathbb{R}^{n_{2} \times n_{2}}$. Systems of the form $M z \equiv(B \otimes C) z=r$ are easy to solve because only $O\left(n^{3 / 2}\right)$ flops are required if $n_{1} \approx n_{2} \approx \sqrt{n}$. To appreciate this point, observe that $(B \otimes C) z=r$ is equivalent to

$$
C Z B^{T}=R
$$

where $Z$ and $R$ are $n_{2}$-by- $n_{1}$ matrices whose columns are segments of the vectors $z$ and $r$ respectively:

$$
\begin{aligned}
& Z(:, k)=z\left((k-1) n_{2}+1: k n_{2}\right) \\
& R(:, k)=r\left((k-1) n_{2}+1: k n_{2}\right) \\
& k=1: n_{1} .
\end{aligned}
$$

(At this point the reader may wish to review the algebra of Kronecker products. See Horn and Johnson(1991) or Van Loan (1992).) If $B$ and $C$ are nonsingular and we apply Gaussian elimination with partial pivoting to produce the 
factorizations $P_{1} B=L_{1} U_{1}$ and $P_{2} C=L_{2} U_{2}$, then $2\left(n_{1}^{3}+n_{2}^{3}\right) / 3$ flops are required. The ensuing multiple triangular system solves involve an additional $2\left(n_{1}^{2} n_{2}+n_{1} n_{2}^{2}\right)$ flops. If $n=n_{1}^{2}=n_{2}^{2}$, then a total of $16 n^{3 / 2} / 3$ flops are needed.

An instructive way to look at the above solution process is to recognize that

$$
\left(P_{1} \otimes P_{2}\right)(B \otimes C)=\left(L_{1} \otimes L_{2}\right)\left(U_{1} \otimes U_{2}\right)
$$

is an $L U$ (with partial pivoting) factorization of $B \otimes C$. This illustrates the adage that a given factorization of $B \otimes C$ can usually be obtained by taking the Kronecker product of the corresponding $B$ and $C$ factorizations :

$$
\begin{aligned}
& \text { Cholesky: } \begin{array}{l}
B=L_{1} L_{1}^{T} \\
C=L_{2} L_{2}^{T}
\end{array} \quad \Rightarrow \quad(B \otimes C)=\left(L_{1} \otimes L_{2}\right)\left(L_{1} \otimes L_{2}\right)^{T} \\
& \text { QR: } \quad \begin{array}{l}
B=Q_{1} R_{1} \\
C=Q_{2} R_{2}
\end{array} \quad \Rightarrow \quad(B \otimes C)=\left(Q_{1} \otimes Q_{2}\right)\left(R_{1} \otimes R_{2}\right)^{T} \\
& \text { SVD: } \quad \begin{array}{l}
B=U_{1} \Sigma_{1} V_{1}^{T} \\
C=U_{2} \Sigma_{2} V_{2}^{T}
\end{array} \quad \Rightarrow \quad(B \otimes C)=\left(U_{1} \otimes U_{2}\right)\left(\Sigma_{1} \otimes \Sigma_{2}\right)\left(V_{1} \otimes V_{2}\right)^{T} \\
& \text { Schur: } \quad \begin{array}{l}
B=U_{1} D_{1} U_{1}^{H} \\
C=U_{2} D_{2} U_{2}^{H}
\end{array} \quad \Rightarrow \quad(B \otimes C)=\left(U_{1} \otimes U_{2}\right)\left(D_{1} \otimes D_{2}\right)\left(U_{1} \otimes U_{2}\right)^{H}
\end{aligned}
$$

Here we are exploiting the fact that

$$
\text { Kronecker products of }\left\{\begin{array}{c}
\text { orthogonal } \\
\text { triangular } \\
\text { diagonal }
\end{array}\right\} \text { matrices are }\left\{\begin{array}{c}
\text { orthogonal } \\
\text { triangular } \\
\text { diagonal }
\end{array}\right\} \text {. }
$$

For a practical illustration of Kronecker product factorizations, see Fausett and Fulton (1992) who apply the idea with QR to solve least squares problems in photogrammetry.

Some factorizations are not "preserved" when Kronecker products are taken:

- A real Schur decomposition of $B \otimes C$ is not obtained by taking the Kronecker product of the real Schur decompositions of $B$ and $C$ because the 2-by-2 bumps in the factors can create "block bumps" in the product. The computational ramifications of this fact are discussed in Bartels and Stewart (1972) and Golub, Nash, and Van Loan (1979).

- If $Q R$ with column pivoting is used to produce the factorizations $B \Pi_{1}=$ $Q_{1} R_{1}$ and $C \Pi_{2}=Q_{2} R_{2}$, then $(B \otimes C)\left(\Pi_{1} \otimes \Pi_{2}\right)=\left(Q_{1} \otimes Q_{2}\right)\left(R_{1} \otimes R_{2}\right)$ is not the factorization rendered by the same algorithm applied to $B \otimes C$.

Despite these anomalies, it is clear that the solution of Kronecker product systems is a nice problem with much structure to exploit. Not only are $O\left(n^{3 / 2}\right)$ solution procedures available, but the form of (1.1) suggests opportunities for using the level-3 BLAS and parallel processing.

The act of finding good preconditioners through an appropriately constrained minimization of $\|A-M\|_{F}$ is not new. For example, Chan (1988) derives a 
useful class of preconditioners for the case when $A$ is Toeplitz by solving

$$
\min _{M \text { circulant }}\|A-M\|_{F} .
$$

Generalizations of this for matrices with Toeplitz blocks are discussed in Chan and Jin (1992).

Our presentation is organized as follows. First, we characterize the optimum Kronecker factors $B$ and $C$ in terms of the singular value decomposition of a permuted version of $A$. Algorithms for determining $B$ and $C$ are discussed is $\S 3$ and $\S 4$. The important cases when $A$ is banded, non-negative, symmetric, and definite are handled in $\S 5$ along with some additional specially structured examples. In $\S 6$ we briefly examine the use of Kronecker product preconditioners.

We conclude this section with a few pointers to related work. The Kronecker product has a long history in mathematics and an excellent review is offered in Henderson, Pukelsheim, and Searle (1983). Computational aspects of the operation are detailed in Pereyra and Scherer (1973) and de Boor (1979).

Kronecker products arise in a number of applied areas. See Andrews and Kane (1970), Swami and Mendel (1990), Brewer (1978), Heap and Lindler (1986), and Rauhala (1980) for Kronecker product discussions of generalized spectra, higher order statistics, systems theory, image processing, and photogrammetry.

In recent years there have been a number of developments that point to an increased role of the Kronecker product in the area of high performance matrix computations. Johnson, Huang, and Johnson (1991) have developed a parallel programming methodology that revolves around the Kronecker product. See also Johnson, Johnson, Rodriguez, and Tolimieri (1990). Regalia and Mitra (1989) and Van Loan (1992) have shown how the organization of fast transforms is clarified through the "language" of Kronecker products.

\section{The Rank-1 Approximation}

Consider the uniform blocking of an $m_{1} m_{2}$-by- $n_{1} n_{2}$ matrix $A$.

$$
A=\left[\begin{array}{cccc}
A_{11} & A_{12} & \cdots & A_{1, n_{1}} \\
A_{21} & A_{22} & \cdots & A_{2, n_{1}} \\
\vdots & \vdots & \ddots & \vdots \\
A_{m_{1}, 1} & A_{m_{1}, 2} & \cdots & A_{m_{1}, n_{1}}
\end{array}\right], \quad A_{i j} \in \mathbb{R}^{m_{2} \times n_{2}}
$$

Using Matlab colon notation, the $(i, j)$ block is given by

$$
A_{i j}=A\left((i-1) m_{2}+1: i m_{2},(j-1) n_{2}+1: j n_{2}\right),
$$

the submatrix defined by rows $(i-1) m_{2}+1$ to $i m_{2}$ and columns $(j-1) n_{2}+1$ to $j n_{2}$. It is not hard to show using the definition of the Kronecker product that

$$
\phi_{A}(B, C)=\sum_{i=1}^{m_{1}} \sum_{j=1}^{n_{1}}\left\|A_{i j}-b_{i j} C\right\|_{F}^{2} .
$$


By keeping the $B$ matrix "intact," we also have

$$
\phi_{A}(B, C)=\sum_{i=1}^{m_{2}} \sum_{j=1}^{n_{2}}\left\|\hat{A}_{i j}-c_{i j} B\right\|_{F}^{2}
$$

where

$$
\hat{A}_{i j}=A\left(i: m_{2}: m, j: n_{2}: n\right)
$$

is the $m_{1}$-by- $n_{1}$ submatrix defined by rows $i, i+m_{2}, i+2 m_{2}, \ldots, i+\left(m_{1}-1\right) m_{2}$ and columns $j, j+n_{2}, j+2 n_{2}, \ldots, j+\left(n_{1}-1\right) n_{2}$. Thinking of matrices at the block level is the key to high performance matrix computations. See Golub and Van Loan (1989).

To proceed further with the analysis of $\phi_{A}(B, C)$, we require the vec operation, which is a way of turning matrices into vectors by "stacking" the columns:

$$
X \in \mathbb{R}^{p \times q} \Rightarrow \operatorname{vec}(X)=\left[\begin{array}{c}
X(1: p, 1) \\
X(1: p, 2) \\
\vdots \\
X(1: p, q)
\end{array}\right] \in \mathbb{R}^{p q}
$$

It turns out that the vec operator can be used to express the minimization of $\|A-B \otimes C\|_{F}^{2}$ as a rank-1 approximation problem. The idea is to rearrange $A$ into another matrix $\tilde{A}$ so that the sum of squares that arise in $\|A-B \otimes C\|_{F}^{2}$ is exactly the same as the sum of squares that arise in $\left\|\tilde{A}-\operatorname{vec}(B) \operatorname{vec}(C)^{T}\right\|_{F}^{2}$. For example, in a 4 -by-4 problem with 2 -by-2 blocks,

$$
\|A-B \otimes C\|_{F}=\left\|\left[\begin{array}{ll|ll}
a_{11} & a_{21} & a_{12} & a_{22} \\
a_{31} & a_{41} & a_{32} & a_{42} \\
\hline a_{13} & a_{23} & a_{14} & a_{24} \\
a_{33} & a_{43} & a_{34} & a_{44}
\end{array}\right]-\left[\begin{array}{l}
b_{11} \\
b_{21} \\
b_{12} \\
b_{22}
\end{array}\right]\left[\begin{array}{llll}
c_{11} & c_{21} & c_{12} & c_{22}
\end{array}\right]\right\|_{F} \text {. }
$$

Refer to the above permuted version of $A$ as $\tilde{A}$. Note that $\tilde{A}$ is not of the form $P A Q$ where $P$ and $Q$ are permutation matrices. Indeed, in our example

- the four rows of $\tilde{A}$ are $v e c$ 's of the 2 -by-2 blocks of $A$ :

$$
A=\left[\begin{array}{ll}
A_{11} & A_{12} \\
A_{21} & A_{22}
\end{array}\right] \Rightarrow \tilde{A}=\left[\begin{array}{c}
\operatorname{vec}\left(A_{11}\right)^{T} \\
\operatorname{vec}\left(A_{21}\right)^{T} \\
\operatorname{vec}\left(A_{12}\right)^{T} \\
\operatorname{vec}\left(A_{22}\right)^{T}
\end{array}\right] .
$$

- the vec's of the 2-by-2 blocks of $\tilde{A}^{T}$ are columns of $A$ :

$$
\tilde{A}=\left[\begin{array}{cc}
\tilde{A}_{11} & \tilde{A}_{12} \\
\tilde{A}_{21} & \tilde{A}_{22}
\end{array}\right] \Rightarrow A=\left[\operatorname{vec}\left(\tilde{A}_{11}^{T}\right)\left|\operatorname{vec}\left(\tilde{A}_{12}^{T}\right)\right| \operatorname{vec}\left(\tilde{A}_{21}^{T}\right) \mid \operatorname{vec}\left(\tilde{A}_{22}^{T}\right)\right] \text {. }
$$

In general, if $m=m_{1} m_{2}, n=n_{1} n_{2}, A \in \mathbb{R}^{m \times n}$, and we have the blocking (2.1), then we define the rearrangement of $A$ (relative to the blocking parameters $m_{1}$, $m_{2}, n_{1}$, and $\left.n_{2}\right)$ by

$$
\mathcal{R}(A)=\left[\begin{array}{c}
A_{1} \\
\vdots \\
A_{n_{1}}
\end{array}\right], \quad A_{j}=\left[\begin{array}{c}
\operatorname{vec}\left(A_{1, j}\right)^{T} \\
\vdots \\
\operatorname{vec}\left(A_{m_{1}, j}\right)^{T}
\end{array}\right], \quad j=1: n_{1} .
$$


Note that $\mathcal{R}(A)$ has $m_{1} n_{1}$ rows and $m_{2} n_{2}$ columns. Thus, $\mathcal{R}(A)$ need not be the same size as $A$. For example, if $m=m_{1} m_{2}=2 \cdot 2$ and $n=n_{1} n_{2}=3 \cdot 2$, then $A$ is 4 -by-6 but

$$
\mathcal{R}(A)=\left[\begin{array}{ll|ll}
a_{11} & a_{21} & a_{12} & a_{22} \\
a_{31} & a_{41} & a_{32} & a_{42} \\
\hline a_{13} & a_{23} & a_{14} & a_{24} \\
a_{33} & a_{43} & a_{34} & a_{44} \\
\hline a_{15} & a_{25} & a_{16} & a_{26} \\
a_{35} & a_{45} & a_{36} & a_{46}
\end{array}\right] .
$$

We are now set to establish a key result that connects the problem of minimizing $\phi_{A}(B, C)$ with the problem of approximating $\tilde{A}$ with a rank-1 matrix.

Theorem 2.1 Assume that $A \in I^{m \times n}$ with $m=m_{1} m_{2}$ and $n=n_{1} n_{2}$. If $B \in \mathbb{R}^{m_{1} \times n_{1}}$ and $C \in I^{m_{2} \times n_{2}}$, then

$$
\|A-B \otimes C\|_{F}=\left\|\mathcal{R}(A)-\operatorname{vec}(B) \operatorname{vec}(C)^{T}\right\|_{F} .
$$

Proof. By applying the vec operator in (2.2) we get:

$$
\begin{aligned}
\|A-B \otimes C\|_{F}^{2} & =\sum_{j=1}^{n_{1}} \sum_{i=1}^{m_{1}}\left\|\operatorname{vec}\left(A_{i j}\right)-b_{i j} \operatorname{vec}(C)\right\|_{2}^{2} \\
& =\sum_{j=1}^{n_{1}} \sum_{i=1}^{m_{1}}\left\|\operatorname{vec}\left(A_{i j}\right)^{T}-b_{i j} \operatorname{vec}(C)^{T}\right\|_{2}^{2} \\
& =\sum_{j=1}^{n_{1}}\left\|A_{j}-B(:, j) \operatorname{vec}(C)^{T}\right\|_{F}^{2} \\
& =\left\|\mathcal{R}(A)-\operatorname{vec}(B) \operatorname{vec}(C)^{T}\right\|_{F}^{2} .
\end{aligned}
$$

The approximation of a given matrix by a rank-1 matrix has a well-known solution in terms of the singular value decomposition.

Corollary 2.2 Assume that $A \in \mathbb{R}^{m \times n}$ with $m=m_{1} m_{2}$ and $n=n_{1} n_{2}$. If $\tilde{A}=\mathcal{R}(A)$ has singular value decomposition

$$
U^{T} \tilde{A} V=\Sigma=\operatorname{diag}\left(\sigma_{i}\right)
$$

where $\sigma_{1}$ is the largest singular value, and $U(:, 1)$ and $V(:, 1)$ are the corresponding singular vectors, then the matrices $B \in \mathbb{R}^{m_{1} \times n_{1}}$ and $C \in \mathbb{R}^{m_{2} \times n_{2}}$ defined by $\operatorname{vec}(B)=\sigma_{1} U(:, 1)$ and $\operatorname{vec}(C)=V(:, 1)$ minimize $\|A-B \otimes C\|_{F}$.

Proof. See Golub and Van Loan(1989, p.73).

The definition (2.4) of $\mathcal{R}(A)$ is in terms of the blocks $A_{i j}$ in (2.1). An alternative characterization can be obtained in terms of the columns of $A$. In particular, we show that

$$
\mathcal{R}(A)=\left[\begin{array}{ccc}
\tilde{A}_{11} & \cdots & \tilde{A}_{1, n_{2}} \\
\vdots & \ddots & \vdots \\
\tilde{A}_{n_{1}, 1} & \cdots & \tilde{A}_{n_{1}, n_{2}}
\end{array}\right]
$$


where $\tilde{A}_{i j} \in \mathbb{R}^{m_{1} \times m_{2}}$ is defined by

$$
\operatorname{vec}\left(\tilde{A}_{i j}^{T}\right)=A\left(:,(i-1) n_{2}+j\right) \quad 1 \leq i \leq n_{1}, 1 \leq j \leq n_{2} .
$$

In view of (2.4) we need only confirm that

$$
A_{i}=\left[\begin{array}{c}
\operatorname{vec}\left(A_{1, i}\right)^{T} \\
\vdots \\
\operatorname{vec}\left(A_{m_{1}, i}\right)^{T}
\end{array}\right]=\left[\tilde{A}_{i, 1}\left|\tilde{A}_{i, 2}\right| \cdots \mid \tilde{A}_{i, n_{2}}\right] .
$$

For $s=1: m_{2}, p=1: n_{2}$, and $q=1: m_{2}$ we have

$$
\left[A_{i}\right]_{s,(p-1) m_{2}+q}=\left[\operatorname{vec}\left(A_{s, i}\right)^{T}\right]_{(p-1) m_{2}+q}=A\left((s-1) m_{2}+q,(i-1) n_{2}+p\right) .
$$

But (2.6) immediately follows because we also have

$$
\left[\tilde{A}_{i, 1}\left|\tilde{A}_{i, 2}\right| \cdots \mid \tilde{A}_{i, n_{2}}\right]_{s,(p-1) m_{2}+q}=\left[\tilde{A}_{i, p}\right]_{s q}=A\left((s-1) m_{2}+q,(i-1) n_{2}+p\right) .
$$

\section{SVD Framework}

The Golub-Reinsch SVD algorithm can be used for computing the largest singular value and corresponding singular vectors of $\mathcal{R}(A)$. However, in view of the potentially large dimension of $\tilde{A}=\mathcal{R}(A)$ in some applications, it may be more appropriate to use the SVD Lanczos process of Golub, Luk, and Overton (1981). Here is how to proceed with the computation of $B \in \mathbb{R}^{m_{1} \times n_{1}}$ and $C \in \mathbb{R}^{m_{2} \times n_{2}}$ :

\section{Framework 1.}

$$
\begin{aligned}
& C=\text { initial guess. } \\
& v_{1} \leftarrow \operatorname{vec}(C) /\|C\|_{F} \\
& p_{0} \leftarrow v_{1} ; \beta_{0} \leftarrow 1 ; j \leftarrow 0 ; u_{0} \leftarrow 0 \\
& \text { while } \beta_{j} \neq 0 \text { (or some other less stringent criteria.) } \\
& v_{j+1} \leftarrow p_{j} / \beta_{j} \\
& j \leftarrow j+1 \\
& r_{j} \leftarrow \tilde{A} v_{j}-\beta_{j-1} u_{j-1} \\
& \alpha_{j} \leftarrow\left\|r_{j}\right\|_{2} \\
& u_{j} \leftarrow r_{j} / \alpha_{j} \\
& p_{j} \leftarrow \tilde{A}^{T} u_{j}-\alpha_{j} v_{j} \\
& \beta_{j} \leftarrow\left\|p_{j}\right\|_{2}
\end{aligned}
$$

There are many subtleties associated with the Lanzcos process and we refer the reader to Cullum and Willoughby(1985) or Golub and Van Loan (1989,p.98ff) for details.

Our only implementation discussion concerns the matrix-vector products $\tilde{A} x$ and $\tilde{A}^{T} x$ that are required by the iteration. The explicit formation of $\mathcal{R}(A)=\tilde{A}$ is not necessary. For example, working with the characterization (2.4), here is a dot product formulation for $y \leftarrow \tilde{A} x$ : 
for $j=1: n_{1}$

for $i=1: m_{1}$

$y\left((j-1) m_{1}+i\right) \leftarrow \operatorname{vec}\left(A_{i j}\right)^{T} x$

end

A saxpy-based procedure for $y \leftarrow \tilde{A}^{T} x$ proceeds as follows:

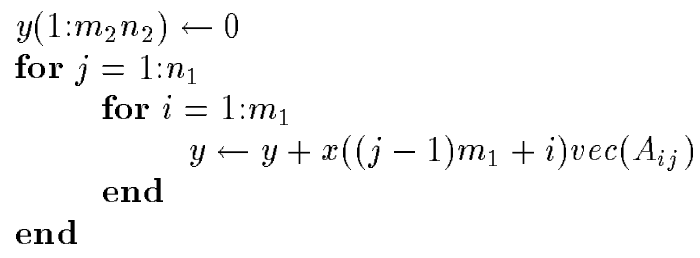

By working with (2.5) we have the following alternative block formulation for $y \leftarrow \tilde{A} x$ :

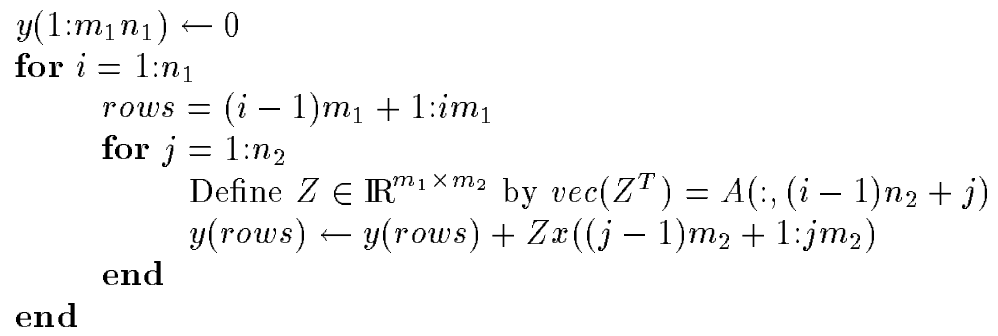

Likewise, we can formulate a procedure for $y \leftarrow \tilde{A}^{T} x$ that is based upon (2.5):

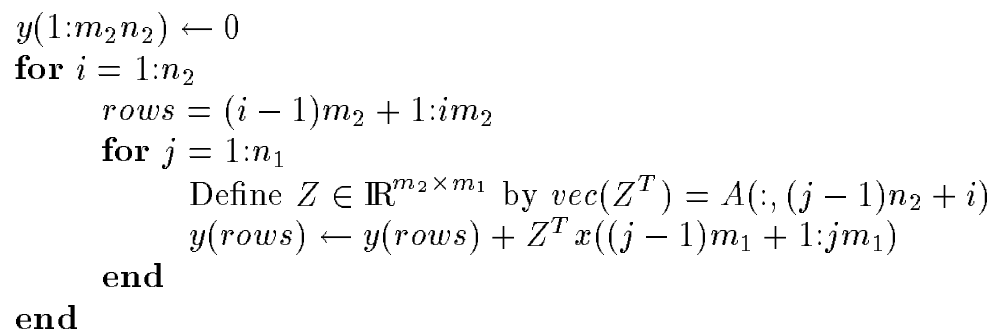

Each of these products requires $2 m_{1} n_{1} m_{2} n_{2}=2 m n$ flops assuming that $\tilde{A}$ is treated as a dense matrix.

\section{The Separable Least Squares Framework}

Note that if we fix $C$, then the problem of minimizing $\phi_{A}(B, C)=\|A-B \otimes C\|_{F}$ is a linear least squares problem with unknowns $b_{i j}$. Likewise, if $B$ is fixed, then the minimization of $\phi_{A}$ is a linear least squares problem in the $c_{i j}$. The following theorem specifies the solution to these linear least squares problems and requires the concept of matrix trace:

$$
X \in \mathbb{R}^{q \times q} \Rightarrow \operatorname{tr}(X)=\sum_{i=1}^{q} x_{i i}
$$


Theorem 4.1 Suppose $m=m_{1} m_{2}, n=n_{1} n_{2}$, and $A \in \mathbb{R} R^{m \times n}$. If $C \in \mathbb{R}^{m_{2} \times n_{2}}$ is fixed, then the matrix $B \in \mathbb{R}^{m_{1} \times n_{1}}$ defined by

$$
b_{i j}=\frac{\operatorname{tr}\left(A_{i j}^{T} C\right)}{\operatorname{tr}\left(C^{T} C\right)} \quad 1 \leq i \leq m_{1}, 1 \leq j \leq n_{1}
$$

minimizes $\|A-B \otimes C\|_{F}$ where $A_{i j}=A\left((i-1) m_{2}+1: i m_{2},(j-1) n_{2}+1: j n_{2}\right)$. Likewise, if $B \in \mathbb{R}^{m_{1} \times n_{1}}$ is fixed, then the matrix $C \in \mathbb{R}^{m_{2} \times n_{2}}$ defined by

$$
c_{i j}=\frac{\operatorname{tr}\left(\hat{A}_{i j}^{T} B\right)}{\operatorname{tr}\left(B^{T} B\right)} \quad 1 \leq i \leq m_{2}, 1 \leq j \leq n_{2}
$$

minimizes $\|A-B \otimes C\|_{F}$ where $\hat{A}_{i j}=A\left(i: m_{2}: m, j: n_{2}: n\right)$.

Proof. Since

$$
\begin{aligned}
\left\|A_{i j}-b_{i j} C\right\|_{F}^{2} & =\operatorname{tr}\left(\left(A_{i j}-b_{i j} C\right)^{T}\left(A_{i j}-b_{i j} C\right)\right) \\
& =\left\|A_{i j}\right\|_{F}^{2}-2 b_{i j} \operatorname{tr}\left(C^{T} A_{i j}\right)+b_{i j}^{2}\|C\|_{F}^{2}
\end{aligned}
$$

it follows from (2.2) that

$$
\frac{\partial \phi_{A}(B, C)}{\partial b_{i j}}=-2 \operatorname{tr}\left(C^{T} A_{i j}\right)+2 b_{i j}\|C\|_{F}^{2} .
$$

Setting all these partials to zero defines the required matrix $B$. The proof of (4.2) is similar.

The above result suggests that we can compute $B$ and $C$ by taking the separable least squares approach of Barham and Drane (1972). The idea is to minimize $\phi_{A}(B, C)$ by alternately improving the $B$ and $C$ matrices through a sequence of linear least squares optimizations:

\section{Framework 2.}

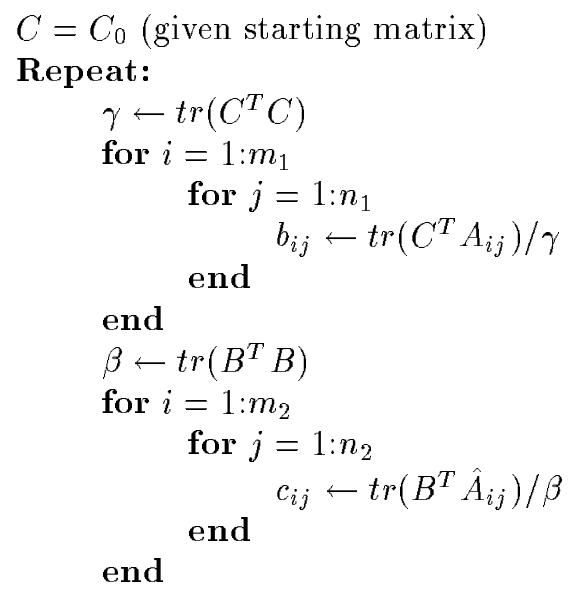

This process requires $4 m_{1} n_{1} m_{2} n_{2}=4 m n$ flops per iteration, the same as Framework 1 . Other methods for nonlinear least squares problems with variables that separate are discussed in Golub and Pereyra (1973) and Kaufman (1975). 
Framework 2 amounts to a power method for the largest singular value of $\tilde{A}=\mathcal{R}(\mathcal{A})$. To see this we switch to "tilde-space" and observe that if

$$
\phi(b, c)=\left\|\tilde{A}-b c^{T}\right\|_{F}^{2} \quad b \in \mathbb{R}^{m_{1} n_{1}}, c \in \mathbb{R}^{m_{2} n_{2}},
$$

then the gradient is given by

$$
\nabla \phi(b, c)=-2\left[\begin{array}{c}
\tilde{A} c-\left(c^{T} c\right) b \\
\tilde{A}^{T} b-\left(b^{T} b\right) c
\end{array}\right] .
$$

If $b$ is fixed, then the minimizing $c$ is obtained by setting $c=\tilde{A}^{T} b / b^{T} b$ for then the $c$-partials are all zero. Likewise, if $c$ is fixed, then the minimizing $b$ is given by $b=\tilde{A} c / c^{T} c$. After $k$ passes through the iteration

$c=c_{0}$ (given starting vector)

Repeat:

$$
\begin{aligned}
& b \leftarrow \tilde{A} c / c^{T} c \\
& c \leftarrow \tilde{A}^{T} b / b^{T} b
\end{aligned}
$$

the vector $c$ is in the direction of $\left(\tilde{A}^{T} \tilde{A}\right)^{k} c_{0}$ and the vector $b$ is in the direction of $\left(\tilde{A} \tilde{A}^{T}\right)^{k-1} \tilde{A} c_{0}$.

The practical implementation of this framework involves all the subtleties that are associated with the power method. See Wilkinson(1965) for a discussion.

\section{Structured Problems}

As we alluded to in $\S 1$, the Kronecker product of two structured matrices is usually structured in the same way:

$$
\text { If } B \text { and } C \text { are }\left\{\begin{array}{l}
\text { banded } \\
\text { non-negative } \\
\text { symmetric } \\
\text { positive definite } \\
\text { stochastic } \\
\text { orthogonal }
\end{array}\right\} \text {, then } B \otimes C \text { is }\left\{\begin{array}{l}
\text { banded } \\
\text { non-negative } \\
\text { symmetric } \\
\text { positive definite } \\
\text { stochastic } \\
\text { orthogonal }
\end{array}\right\} \text {. }
$$

We are interested in the structure of the solution to the Kronecker approximation problem given that $A$ is structured. In the following subsections we use Corollary 2.2 and Theorem 4.1 to establish a number results about structured problems.

\subsection{Bandedness}

We first show how bandedness in $A$ "shows up" in $B$ and $C$.

Theorem 5.1 Suppose $n=n_{1} n_{2}, A \in I R^{n \times n}$ has bandwidth $p n_{2}$, and that each block in

$$
A=\left[\begin{array}{ccc}
A_{11} & \cdots & A_{1, n_{1}} \\
\vdots & \ddots & \vdots \\
A_{n_{1}, 1} & \cdots & A_{n_{1} n_{1}}
\end{array}\right] \quad A_{i j} \in \mathbb{R}^{n_{2} \times n_{2}}
$$

has bandwidth $q$ or less. If $B \in \mathbb{R}^{n_{1} \times n_{1}}$ and $C \in \mathbb{R}^{n_{2} \times n_{2}}$ minimize $\|A-B \otimes C\|_{F}$, then $B$ has bandwidth $p$ and $C$ has bandwidth $q$. 
Proof. Since $A$ has bandwidth $p n_{2}$, it follows that $A_{i j}=0$ if $|i-j|>p$. From (2.2) we have $b_{i j}=0$ whenever $|i-j|>p$. Since each $A_{i j}$ has bandwidth $q$, it follows that the minimization of $\left\|A_{i j}-b_{i j} C\right\|_{F}$ requires setting $c_{r s}$ to zero whenever $|r-s|>q$. Thus, a minimizing $C$ must have bandwidth $q$.

\subsection{Non-Negativity}

We first show that if $A$ and $C$ are non-negative, then the $B$ that minimizes $\phi_{A}(B, C)$ is also non-negative.

Theorem 5.2 If $m=m_{1} m_{2}, n=n_{1} n_{2}, A \in \mathbb{R}^{m \times n}$, and $C \in \mathbb{R}^{m_{2} \times n_{2}}$ are nonnegative, then there exists a non-negative $B \in \mathbb{R}^{m_{1} \times n_{1}}$ that minimizes $\|A-B \otimes C\|_{F}$.

Proof. Using the non-negativity of $C$ and Theorem 4.1,

$$
b_{i j}=\frac{\operatorname{tr}\left(A_{i j}^{T} C\right)}{\operatorname{tr}\left(C^{T} C\right)} \geq 0
$$

for $i=1: m_{1}$ and $j=1: n_{1}$.

In the same way, we can show that if $A$ and $B$ are non-negative, then the $C$ that minimizes $\|A-B \otimes C\|$ is also non-negative. Thus, if we start with a non-negative $C$ in Framework 2, then all subsequent $B$ and $C$ matrices are nonnegative. The following theorem shows that this restriction poses no difficultly because the optimum $B$ and $C$ are also non-negative.

Theorem 5.3 If $m=m_{1} m_{2}, n=n_{1} n_{2}$, and $A \in \mathbb{R}^{m \times n}$ is non-negative, then there exist non-negative matrices $B \in \mathbb{R}^{m_{1} \times n_{1}}$ and $C \in \mathbb{R}^{m_{2} \times m_{2}}$ such that $\|A-B \otimes C\|_{F}$ is minimized.

Proof. Note that $\tilde{A}=\mathcal{R}(A)$ has non-negative entries and let $\sigma_{1}$ be its largest singular value. Peron-Frobenius theory tells us that there exist non-negative $u \in \mathbb{R}^{m_{1} n_{1}}$ and $v \in \mathbb{R}^{m_{2} n_{2}}$ so that $\tilde{A}^{T} \tilde{A} v=\sigma_{1}^{2} v$ and $\tilde{A} \tilde{A}^{T} u=\sigma_{1}^{2} u$. (See Horn and Johnson (1985,p. 503). But $u$ and $v$ are the right and left singular vectors of $\tilde{A}$ and so the matrices $B$ and $C$ as specified in Corollary 2.2 are nonnegative.

\subsection{Symmetry}

Turning next to the issue of symmetry, we show that if $A$ and $C$ are symmetric, then a symmetric $B$ can be found to minimize $\phi_{A}(B, C)$.

Theorem 5.4 If $n=n_{1} n_{2}, A \in \mathbb{R}^{n \times n}$ and $C \in \mathbb{R}^{n_{2} \times n_{2}}$ are symmetric, then there exists a symmetric $B \in \mathbb{R}^{n_{1} \times n_{1}}$ that minimizes $\|A-B \otimes C\|_{F}$.

Proof. Since $A$ is symmetric, $A_{j i}=A_{i j}^{T}$. Using elementary properties of the trace we have

$$
b_{i j}=\frac{\operatorname{tr}\left(A_{i j}^{T} C\right)}{\operatorname{tr}\left(C^{T} C\right)}=\frac{\operatorname{tr}\left(A_{j i} C\right)}{\operatorname{tr}\left(C^{T} C\right)}=\frac{\operatorname{tr}\left(C A_{j i}\right.}{\operatorname{tr}\left(C^{T} C\right)}=\frac{\operatorname{tr}\left(A_{j i}^{T} C\right)}{\operatorname{tr}\left(C^{T} C\right)}=b_{j i}
$$

for all $1 \leq i, j \leq n_{1}$. It follows that $B$ is symmetric.

It is equally straightforward to establish that a symmetric $C$ can be found to minimize $\|A-B \otimes C\|_{F}$ is $A$ and $B$ are symmetric.

Analogous results are applicable if the "frozen factor" is skew-symmetric: 
Theorem 5.5 If $n=n_{1} n_{2}, A \in \mathbb{R}^{n \times n}$ is symmetric and $C \in \mathbb{R}^{n_{2} \times n_{2}}$ is skewsymmetric, then there exists a skew-symmetric $B \in \mathbb{R}^{n_{1} \times n_{1}}$ that minimizes $\|A-B \otimes C\|_{F}$.

Proof.

$$
b_{i j}=\frac{\operatorname{tr}\left(A_{i j}^{T} C\right)}{\operatorname{tr}\left(C^{T} C\right)}=-\frac{\operatorname{tr}\left(A_{j i}^{T} C\right)}{\operatorname{tr}\left(C^{T} C\right)}=-b_{j i} .
$$

The optimum Kronecker approximation of a symmetric matrix may have skew-symmetric factors as consideration of the following example shows:

$$
A=\left[\begin{array}{rrrr}
0 & 0 & 0 & 1 \\
0 & 0 & -1 & 0 \\
0 & -1 & 0 & 0 \\
1 & 0 & 0 & 0
\end{array}\right]=\left[\begin{array}{rr}
0 & 1 \\
-1 & 0
\end{array}\right] \otimes\left[\begin{array}{rr}
0 & 1 \\
-1 & 0
\end{array}\right] \text {. }
$$

For this particular $A$, it is not possible to find symmetric $B$ and $C$ for which we have $A=B \otimes C$. The following theorem summarizes the situation.

Theorem 5.6 Suppose $n=n_{1} n_{2}$ and $A \in R^{n \times n}$ is symmetric. If $\|A-B \otimes C\|_{F}$ cannot minimized by symmetric matrices $B \in \mathbb{R}^{n_{1} \times n_{1}}$ and $C \in \mathbb{R}^{n_{2} \times n_{2}}$, then it can be minimized by skew-symmetric matrices $B \in I^{n_{1} \times n_{1}}$ and $C \in I R^{n_{2} \times n_{2}}$.

Proof. For any positive integer $q$, define the following orthogonal subspaces of $\mathbb{R}^{q^{2}}:$

$$
\begin{aligned}
& S_{+}^{(q)}=\left\{x \in \mathbb{R}^{q^{2}}: x=\operatorname{vec}(X) \text { for some symmetric } X \in \mathbb{R}^{q \times q}\right\} \\
& S_{-}^{(q)}=\left\{x \in \mathbb{R}^{q^{2}}: x=\operatorname{vec}(X) \text { for some skew-symmetric } X \in \mathbb{R}^{q \times q}\right\}
\end{aligned}
$$

Note that $\mathbb{R}^{q^{2}}=S_{+}^{(q)} \oplus S_{-}^{(q)}$.

Now suppose that $y=\mathcal{R}(A) x$ and that $X \in \mathbb{R}^{n_{2} \times n_{2}}$ and $Y \in \mathbb{R}^{n_{1} \times n_{1}}$ are defined by $x=\operatorname{vec}(X)$ and $y=\operatorname{vec}(Y)$, respectively. From (2.1) we know that

$$
[Y]_{i j}=\operatorname{vec}\left(A_{i j}\right)^{T} x=\operatorname{tr}\left(A_{i j}^{T} X\right) \quad 1 \leq i, j \leq n_{1} .
$$

If $x \in S_{+}^{\left(n_{2}\right)}$, then since $A$ is symmetric we have

$$
[Y]_{i j}-[Y]_{j i}=\operatorname{tr}\left(\left(A_{i j}^{T}-A_{j i}^{T}\right) X\right)=\operatorname{tr}\left(\left(A_{i j}^{T}-A_{i j}\right) X\right)=\operatorname{vec}\left(A_{i j}^{T}-A_{i j}\right)^{T} x=0
$$

since $\operatorname{vec}\left(A_{i j}^{T}-A_{i j}\right) \in S_{-}^{\left(n_{2}\right)}$. Thus,

$$
x \in S_{+}^{\left(n_{2}\right)} \Rightarrow \mathcal{R}(A) x \in S_{+}^{\left(n_{1}\right)}
$$

Likewise,

$$
x \in S_{-}^{\left(n_{2}\right)} \Rightarrow \mathcal{R}(A) x \in S_{-}^{\left(n_{1}\right)}
$$

Thus, $\left(S_{+}^{\left(n_{2}\right)}, S_{+}^{\left(n_{1}\right)}\right)$ and $\left(S_{-}^{\left(n_{2}\right)}, S_{-}^{\left(n_{1}\right)}\right)$ are singular subspace pairs for $\mathcal{R}(A)$. It follows that the largest singular value and corresponding singular vectors must be associated with one of these pairs.

Theorem 5.6 can also be established by observing that if $A$ is symmetric, then

$$
P_{n_{1}} \mathcal{R}(A) P_{n_{2}}^{T}=\mathcal{R}(A)
$$


where $P_{q}$ designates the vec permutation matrix on $\mathbb{R}^{q^{2}}$ :

$$
P_{q} \operatorname{vec}(X)=\operatorname{vec}\left(X^{T}\right) \quad X \in \mathbb{R}^{q \times q} .
$$

This permutation connects the vec of a matrix and the vec of its transpose. See Henderson and Searle (1981) for further details.

\subsection{Positive Definiteness}

We first show that if the initial guess matrix in Framework 2 is positive definite, then all subsequent $B$ and $C$ iterates are positive definite.

Theorem 5.7 If $n=n_{1}^{2}, A \in \mathbb{R}^{n \times n}$ and $C \in \mathbb{R}^{n_{2} \times n_{2}}$ are symmetric positive definite, then there exists a symmetric positive definite $B \in \mathbb{R}^{n_{1} \times n_{1}}$ that minimizes $\phi_{A}(B, C)$. Likewise, if $B \in \mathbb{R}^{n_{1} \times n_{1}}$ is symmetric positive definite, then there exists a symmetric positive definite $C \in \mathbb{R}^{n_{2} \times n_{2}}$ that minimizes $\phi_{A}(B, C)$.

Proof. If each entry $b_{i j}$ in $B \in \mathbb{R}^{n_{1} \times n_{1}}$ satisfies $b_{i j}=\operatorname{tr}\left(C^{T} A_{i j}\right) / \operatorname{tr}\left(C^{T} C\right)$, and if $y \in \mathbb{R}^{n_{1}}$, then using the linearity of the trace we have

$$
y^{T} B y=\sum_{i=1}^{n_{1}} \sum_{j=1}^{n_{1}} b_{i j} y_{i} y_{j}=\sum_{i=1}^{n_{1}} \sum_{j=1}^{n_{1}} y_{i} y_{j} \operatorname{tr}\left(C^{T} A_{i j}\right) / \operatorname{tr}\left(C^{T} C\right)=\operatorname{tr}\left(C^{T} \hat{A}\right)
$$

where

$$
\hat{A}=\sum_{i=1}^{n_{1}} \sum_{j=1}^{n_{1}} y_{i} y_{j} A_{i j}
$$

The matrix $\hat{A}$ is positive definite because for any $z \in \mathbb{R}^{n_{1}}$ we have

$$
0<(z \otimes y)^{T} A(z \otimes y)=\left[z_{1} y^{T}|\cdots| z_{n_{1}} y^{T}\right]\left[A_{i j}\right]\left[\begin{array}{c}
z_{1} y \\
\vdots \\
z_{n_{1}} y
\end{array}\right]=z^{T} \hat{A} z
$$

Since $C$ is positive definite, it has a Cholesky factorization $C=L L^{T}$. From (5.1) and the fact that the trace is invariant under similarity transformations, gives

$$
y^{T} B y=\operatorname{tr}\left(C^{T} \hat{A}\right)=\operatorname{tr}\left(L L^{T} \hat{A}\right)=\operatorname{tr}\left(L^{-1}\left(L L^{T} \hat{A}\right) L\right)=\operatorname{tr}\left(L^{T} \hat{A} L\right)>0 .
$$

The proof that $C$ is positive definite when $B$ is given is similar.

The next result shows that if $A$ is symmetric and positive definite, then the same can be said about the optimum $B$ and $C$.

Theorem 5.8 If $n=n_{1} n_{2}$ and $A \in \mathbb{R}^{n \times n}$ is symmetric positive definite, then there exists symmetric positive definite $B \in \mathbb{R}^{n_{1} \times n_{1}}$ and $C \in \mathbb{R}^{n_{2} \times n_{2}}$ that minimize $\phi_{A}(B, C)$.

Proof. ¿From Theorem 5.6 we may select the optimum $B$ and $C$ to be either both skew-symmetric or both symmetric. We first show that the latter must be the case. 
If $B$ is skew-symmetric, then there exists a real orthogonal $U_{B}$ such that

$$
U_{B}^{T} B U_{B}=B_{1}
$$

where $B_{1}$ is a direct sum of 1 -by-1 and 2 -by-2 skew-symmetric blocks. The 1-by-1's are (of course) zero and the 2-by-2's have the form

$$
M=\left[\begin{array}{rr}
0 & m \\
-m & 0
\end{array}\right]
$$

and correspond to the complex conjugate eigenpairs of $B$. The decomposition (5.2) is just the real Schur decomposition. Note that the unitary matrix

$$
Z=\frac{1}{\sqrt{2}}\left[\begin{array}{ll}
1 & i \\
i & 1
\end{array}\right]
$$

diagonalizes $M$ :

$$
Z^{H} M Z=\left[\begin{array}{cc}
i m & 0 \\
0 & -i m
\end{array}\right]
$$

Let $V_{B}$ be the unitary matrix that has copies of $Z$ on the diagonal which correspond to the 2 -by-2 blocks in $B_{1}$, and which is the identity elsewhere. It follows that

$$
V_{B}^{H} U_{B}^{T} B U_{B} V_{B}=D_{B}
$$

is diagonal. Let us refer to this decomposion as the structured Schur decomposition of $B$. Assume that $C$ is also skew-symmetric and let

$$
V_{C}^{H} U_{C}^{T} C U_{C} V_{C}=D_{C}
$$

be its structured Schur decomposition. For a matrix $H$, let $|H|$ be the matrix obtained by taking the absolute values of each entry. Since

$$
Z\left|\left[\begin{array}{cc}
i m & 0 \\
0 & -i m
\end{array}\right]\right| Z^{H}=|m| I_{2}
$$

it is easy to check that the matrices

$$
\begin{aligned}
& B_{+}=U_{B} V_{B}\left|D_{B}\right| V_{B}^{H} U_{B}^{T} \\
& C_{+}=U_{C} V_{C}\left|D_{C}\right| V_{C}^{H} U_{C}^{T}
\end{aligned}
$$

are real and symmetric.

Let $Q=Q_{B} \otimes Q_{C}$ where $Q_{B}=U_{B} V_{B}$ and $Q_{C}=U_{C} V_{C}$. Define the off operation on matrices as follows:

$$
o f f(M)=\sum_{i \neq j} m_{i j}^{2} .
$$

Setting $D_{A}$ to be the diagonal part of $Q^{H} A Q$, we see that

$$
\begin{aligned}
\left\|A-B_{+} \otimes C_{+}\right\|_{F}^{2} & =\left\|Q^{H} A Q-\left|D_{B}\right| \otimes\left|D_{C}\right|\right\|_{F}^{2} \\
& =\operatorname{off}\left(Q^{H} A Q\right)+\left\|D_{A}-\left|D_{B}\right| \otimes\left|D_{C}\right|\right\|_{F}^{2}
\end{aligned}
$$


while

$$
\begin{aligned}
\|A-B \otimes C\|_{F}^{2} & =\left\|Q^{H} A Q-D_{B} \otimes D_{C}\right\|_{F}^{2} \\
& =\operatorname{off}\left(Q^{H} A Q\right)+\left\|D_{A}-D_{B} \otimes D_{C}\right\|_{F}^{2} .
\end{aligned}
$$

Since $Q^{H} A Q$ is positive definite, $D_{A}$ has positive diagonal entries. Moreover, $D_{B} \otimes D_{C}$ is a real diagonal matrix with some negative diagonal entries. It follows that

$$
\left\|D_{A}-\left|D_{B}\right| \otimes\left|D_{C}\right|\right\|_{F}^{2}<\left\|D_{A}-D_{B} \otimes D_{C}\right\|_{F}^{2} .
$$

and so

$$
\left\|A-B_{+} \otimes C_{+}\right\|_{F}<\|A-B \otimes C\|_{F} .
$$

This shows that a skew-symmetric pair cannot minimize $\phi_{A}(B, C)$.

Knowing now that the optimizing $B$ and $C$ are symmetric, it remains for us to show that they are both positive definite. Suppose

$$
\begin{aligned}
& Q_{1}^{T} B Q_{1}=D_{1}=\operatorname{diag}\left(\lambda_{1}, \ldots, \lambda_{n_{1}}\right) \\
& Q_{2}^{T} C Q_{2}=D_{2}=\operatorname{diag}\left(\mu_{1}, \ldots, \mu_{n_{2}}\right)
\end{aligned}
$$

are Schur decompositions. Set $Q=Q_{1} \otimes Q_{2}$ and let $D=\operatorname{diag}\left(d_{1}, \ldots, d_{n}\right)$ be the diagonal part of $F=Q^{T} A Q$. Thus,

$$
\begin{aligned}
\|A-B \otimes C\|_{F}^{2} & =\left\|Q^{T}(A-B \otimes C) Q\right\|_{F}^{2} \\
& =\left\|F-D_{1} \otimes D_{2}\right\|_{F}^{2}=\left\|D-D_{1} \otimes D_{2}\right\|_{F}^{2}+\text { off }(F) .
\end{aligned}
$$

Note that

$$
\left\|D-D_{1} \otimes D_{2}\right\|_{F}^{2}=\sum_{i=1}^{n_{1}} \sum_{j=1}^{n_{2}}=\left(d_{(i-1) n_{2}+j}-\lambda_{i} \mu_{j}\right)^{2} .
$$

Since $D$ has positive diagonal entries and

$$
\left(d_{(i-1) n_{2}+j}-\lambda_{i} \mu_{j}\right)^{2}-\left(d_{(i-1) n_{2}+j}-\left|\lambda_{i} \mu_{j}\right|\right)^{2}=\left|\lambda_{i}\right|^{2}\left|\mu_{j}\right|^{2}-\lambda_{i}^{2} \mu_{j}^{2},>0,
$$

it follows that the $\lambda_{i}$ and $\mu_{j}$ should all have the same sign. Otherwise, $B$ and $C$ will not render the minimum sum of squares. Since $\phi_{A}(-B,-C)=\phi_{A}(B, C)$, we may assume without loss of generality that this sign is positive. This implies that symmetric positive definite $B$ and $C$ may be chosen to be minimize $\phi_{A}(B, C)$.

\subsection{Sums of Kronecker Products}

Next, we consider the situation when the matrix $A$ to be approximated is a sum of Kronecker products:

$$
A=\sum_{i=1}^{p}\left(G_{i} \otimes F_{i}\right) .
$$

Assume that each $G_{i}$ is $m_{1}$-by- $n_{1}$ and each $F_{i}$ is $m_{2}$-by- $n_{2}$. It follows that if $f_{i}=\operatorname{vec}\left(F_{i}\right)$ and $g_{i}=\operatorname{vec}\left(G_{i}\right)$, then

$$
\tilde{A}=\mathcal{R}(A)=\sum_{i=1}^{p} \mathcal{R}\left(G_{i} \otimes F_{i}\right)=\sum_{i=1}^{p} g_{i} f_{i}^{T}
$$


is a rank- $p$ matrix. This has two important ramifications. First, it means that matrix-vector products of the form $\tilde{A} x$ and $\tilde{A}^{T} x$ cost $O((m+n) p)$ flops where $m=m_{1} m_{2}$ and $n=n_{1} n_{2}$. Second, it means that the optimum $B$ and $C$ are linear combinations of the $G_{i}$ and $F_{i}$ :

$$
\begin{aligned}
B & =\alpha_{1} G_{1}+\cdots+\alpha_{p} G_{p} \\
C & =\beta_{1} F_{1}+\cdots+\beta_{p} F_{p}
\end{aligned}
$$

The problem of approximating matrices of the form $(I \otimes F)+(G \otimes I)$ is discussed further in $\S 6$.

\subsection{Approximation with Linear Homogeneous Constraints}

Consider the problem of approximating $A$ with a Kronecker product $B \otimes C$ that has a prescribed structure. If the constraints on $B$ and $C$ are linear and homogeneous, then we are looking at a problem with the following form:

$$
\begin{aligned}
\min & \|A-B \otimes C\|_{F} . \\
S_{1}^{T} \operatorname{vec}(B) & =0 \\
S_{2}^{T} \operatorname{vec}(C) & =0
\end{aligned}
$$

Here, $A \in \mathbb{R}^{m \times n}, m=m_{1} m_{2}, n=n_{1} n_{2}, B \in \mathbb{R}^{m_{1} \times n_{1}}, C \in \mathbb{R}^{m_{2} \times n_{2}}, S_{1} \in \mathbb{R}^{m_{1} n_{1} \times p_{1}}$, $S_{2} \in \mathbb{R}^{m_{2} n_{2} \times p_{2}}$, and we assume that $S_{1}$ and $S_{2}$ have full column rank. By choosing these constraint matrices properly, we can force $B$ and $C$ to take on any prescribed sparsity pattern. Circulant, Toeplitz, Hankel, and Hamiltonian structures can also be imposed.

To solve the constrained problem we follow the techniques espoused in Golub (1973) where various modified eigenvalue problems are discussed. Let $b=$ $\operatorname{vec}(B), c=\operatorname{vec}(C)$, and assume that we have the $\mathrm{QR}$ factorizations

$$
S_{1}=Q_{1}\left[\begin{array}{c}
R_{1} \\
0
\end{array}\right] \quad S_{2}=Q_{2}\left[\begin{array}{c}
R_{2} \\
0
\end{array}\right]
$$

where $R_{1}$ and $R_{2}$ are square. If

$$
Q_{1}^{T} \mathcal{R}(A) Q_{2}=\left[\begin{array}{cc}
\tilde{A}_{11} & \tilde{A}_{12} \\
\tilde{A}_{21} & \tilde{A}_{22}
\end{array}\right], \quad Q_{1}^{T} b=\left[\begin{array}{l}
b_{1} \\
b_{2}
\end{array}\right], \quad Q_{2}^{T} c=\left[\begin{array}{l}
c_{1} \\
c_{2}
\end{array}\right]
$$

are partitioned conformably with (5.4), then (5.3) transforms to the problem of minimizing

$$
\left\|\left[\begin{array}{ll}
\tilde{A}_{11} & \tilde{A}_{12} \\
\tilde{A}_{21} & \tilde{A}_{22}
\end{array}\right]-\left[\begin{array}{l}
b_{1} \\
b_{2}
\end{array}\right]\left[\begin{array}{l}
c_{1} \\
c_{2}
\end{array}\right]^{T}\right\|_{F}
$$

subject to the constraints

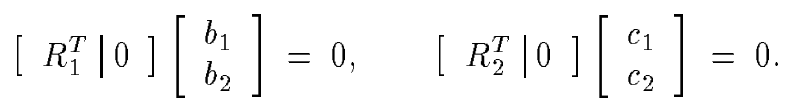

It follows that $b_{1}$ and $c_{1}$ are both zero and that the optimum $b_{2}$ and $c_{2}$ can be obtained by solving the unconstrained problem

$$
\min \left\|\tilde{A}_{22}-b_{2} c_{2}^{T}\right\|_{F} \text {. }
$$

Collecting results, we see that $B$ and $C$ are prescribed by

$$
\operatorname{vec}(B)=Q_{1}\left[\begin{array}{c}
0 \\
b_{2}
\end{array}\right], \quad \operatorname{vec}(C)=Q_{2}\left[\begin{array}{c}
0 \\
c_{2}
\end{array}\right] .
$$




\subsection{Stochastic and Orthogonal Problems}

The non-negative matrix $A \in \mathbb{R}^{n \times n}$ is stochastic if $e_{n}^{T} A=e_{n}^{T}$ where $e_{n}$ is the $n$-vector of ones. If $n=n_{1} n_{2}$ and $B \in \mathbb{R}^{n_{1} \times n_{1}}$ and $C \in \mathbb{R}^{n_{2} \times n_{2}}$ minimize $\phi_{A}(B, C)$, then it does not follow that $B$ and $C$ are stochastic. For example, if

$$
A=\left[\begin{array}{llll}
.1 & .5 & .2 & .6 \\
.4 & .1 & .1 & .2 \\
.2 & .0 & .3 & .1 \\
.3 & .4 & .4 & .1
\end{array}\right]
$$

then, after normalizing $B$ and $C$ so that $b_{11}+b_{21}=1$ we have

$$
B=\left[\begin{array}{ll}
.6228 & .5939 \\
.3772 & .4298
\end{array}\right] \quad C=\left[\begin{array}{ll}
.3610 & .6657 \\
.5560 & .3512
\end{array}\right]
$$

Note that $B$ and $C$ are not quite stochastic. Thus, to get the best stochastic Kronecker product approximation we must apply a constrained nonlinear least squares solver to the problem

$$
\begin{aligned}
& \min _{e_{n_{1}}^{T} B}=e_{n_{1}}^{T}, B \geq 0 \\
& e_{n_{2}}^{T} C=e_{n_{2}}^{T}, C \geq 0
\end{aligned}
$$

Another structured problem that is not solvable by our SVD framework is the case when $A$ is orthogonal and we insist that the optimizing $B$ and $C$ be orthogonal. It does not follow that orthogonal $B$ and $C$ minimize $\phi_{A}(B, C)$. Thus, we are led to another constrained nonlinear leasts squares problem:

$$
\begin{aligned}
& \min _{B^{T} B=I_{n_{1}}}\|A-B \otimes C\|_{F} . \\
& C^{T} C=I_{n_{2}}
\end{aligned}
$$

A reasonable initial guess $\left(B_{0}, C_{0}\right)$ in this setting is to set $B_{0}$ and $C_{0}$ to be the closest orthogonal matrices to the $B$ and $C$ that minimize $\phi_{A}(B, C)$.

\section{Kronecker Product Preconditioners}

To acquire some intuition about the use of Kronecker products as pre-conditioners, consider the $A x=b$ problem where

$$
A=a_{1}\left(I_{n_{1}} \otimes I_{n_{2}}\right)+a_{2}\left(I_{n_{1}} \otimes J_{n_{2}}\right)+a_{2}\left(J_{n_{1}} \otimes I_{n_{2}}\right)+a_{3}\left(J_{n_{1}} \otimes J_{n_{2}}\right),
$$

$n=n_{1} n_{2}$, and $J_{m}$ is the $m$-by- $m$ symmetric tridiagonal matrix

$$
J_{m}=\left[\begin{array}{ccccc}
0 & 1 & 0 & \cdots & 0 \\
1 & 0 & 1 & \cdots & 0 \\
\vdots & \vdots & \ddots & & \vdots \\
0 & 0 & \cdots & 0 & 1 \\
0 & 0 & \cdots & 1 & 0
\end{array}\right]
$$


Matrices with this structure arise in many applications. For example, the usual discretization of Poisson's equation on a rectangle with the "Dirichlet stencil"

\begin{tabular}{|l|l|l|}
\hline$a_{3}$ & $a_{2}$ & $a_{3}$ \\
\hline$a_{2}$ & $a_{1}$ & $a_{2}$ \\
\hline$a_{3}$ & $a_{2}$ & $a_{3}$ \\
\hline
\end{tabular}$=$\begin{tabular}{|r|r|r|}
\hline 0 & -1 & 0 \\
\hline-1 & 4 & -1 \\
\hline 0 & -1 & 0 \\
\hline
\end{tabular}

leads to

$$
A=\left(2 I_{n_{1}}-J_{n_{1}}\right) \otimes I_{n_{2}}+I_{n_{1}} \otimes\left(2 I_{n_{2}}-J_{n_{2}}\right) .
$$

In computer vision, the Laplace stencil defined by

\begin{tabular}{|l|l|l|}
\hline$a_{3}$ & $a_{2}$ & $a_{3}$ \\
\hline$a_{2}$ & $a_{1}$ & $a_{2}$ \\
\hline$a_{3}$ & $a_{2}$ & $a_{3}$ \\
\hline
\end{tabular}$=$\begin{tabular}{|r|r|r|}
\hline-1 & -4 & -1 \\
\hline-4 & 20 & -4 \\
\hline-1 & -4 & -1 \\
\hline
\end{tabular}

is frequently used, see Klaus and Horn (1990). This leads to

$$
A=\left(2 I_{n_{1}}-J_{n_{1}}\right) \otimes\left(5 I_{n_{2}}+\frac{1}{2} J_{n_{2}}\right)+\left(5 I_{n_{1}}+\frac{1}{2} J_{n_{1}}\right) \otimes\left(2 I_{n_{2}}-J_{n_{2}}\right) .
$$

In general, if we define the constants

$$
\begin{aligned}
& \alpha_{1}=2, \quad \alpha_{2}=2\left(a_{2}-\sqrt{a_{2}^{2}-a_{1} a_{3}}\right) / a_{1}, \\
& \beta_{1}=a_{1} / 4, \quad \beta_{2}=\left(a_{2}+\sqrt{a_{2}^{2}-a_{1} a_{3}}\right) / 4,
\end{aligned}
$$

then the matrix $A$ in (6.1) can be expressed in the form

$$
A=\left(\alpha_{1} I_{n_{1}}+\alpha_{2} J_{n_{1}}\right) \otimes\left(\beta_{1} I_{n_{2}}+\beta_{2} J_{n_{2}}\right)+\left(\beta_{1} I_{n_{1}}+\beta_{2} J_{n_{1}}\right) \otimes\left(\alpha_{1} I_{n_{2}}+\alpha_{2} J_{n_{2}}\right) .
$$

Thus, $A$ is the sum of two Kronecker products and the remarks made in $§ 5.5$ apply. Since the rank of $\tilde{A}$ is two, the singular vectors that define the optimal $B$ and $C$ can be computed in $O(n)$ flops. These matrices are tridiagonal, symmetric, and positive definite in view of the discussions in $\S 5$.

Let us focus on the case when $A$ is given by (6.2). For simplicity, define the $[-12-1]$ tridiagonal matrix

$$
T_{m}=2 I_{m}-J_{m}
$$

and note that

$$
A=T_{n_{1}} \otimes I_{n_{2}}+I_{n_{1}} \otimes T_{n_{2}} .
$$

¿From $\S 5.5$ we know that the optimizing $B$ and $C$ have the form

$$
\begin{aligned}
& B=b_{1} I_{n_{1}}+b_{2} T_{n_{1}} \\
& C=c_{1} I_{n_{2}}+c_{2} T_{n_{2}} .
\end{aligned}
$$

The matrix $T_{m}$ has known eigenvalues:

$$
Q_{m}^{T} T_{m} Q_{m}=D_{m}=\operatorname{diag}\left(\lambda_{1}^{(m)}, \ldots, \lambda_{m}^{(m)}\right), \quad \lambda_{j}^{(m)}=4 \sin ^{2}\left(\frac{j \pi}{2(m+1)}\right) .
$$


Using this result, it can be shown that the Kronecker approximation problem involves choosing $b_{1}, b_{2}, c_{1}$, and $c_{2}$ so that

$$
\begin{aligned}
& \|A-B \otimes C\|_{F}^{2}= \\
= & \left\|\left(T_{n_{1}} \otimes I_{n_{2}}+I_{n_{1}} \otimes T_{n_{2}}\right)-\left(b_{1} I_{n_{1}}+b_{2} T_{n_{1}}\right) \otimes\left(c_{1} I_{n_{2}}+c_{2} T_{n_{2}}\right)\right\|_{F}^{2} \\
= & \left\|\left(D_{n_{1}} \otimes I_{n_{2}}+I_{n_{1}} \otimes D_{n_{2}}\right)-\left(b_{1} I_{n_{1}}+b_{2} D_{n_{1}}\right) \otimes\left(c_{1} I_{n_{2}}+c_{2} D_{n_{2}}\right)\right\|_{F} \\
= & \sum_{i=1}^{n_{1}} \sum_{j=1}^{n_{2}}\left[\left(\lambda_{i}^{\left(n_{1}\right)}+\lambda_{j}^{\left(n_{2}\right)}\right)-\left(b_{1}+b_{2} \lambda_{i}^{\left(n_{1}\right)}\right)\left(c_{1}+c_{2} \lambda_{j}^{\left(n_{2}\right)}\right)\right]^{2}
\end{aligned}
$$

is minimized. The eigenvalue distribution of $M^{-1} A$, which is crucial to the success of $M=B \otimes C$ as a preconditioner, can also be examined in closed form once $b_{1}, b_{2}, c_{1}$, and $c_{2}$ are known:

$$
\lambda_{i j}\left(M^{-1} A\right)=\frac{\lambda_{i}^{\left(n_{1}\right)}+\lambda_{j}^{\left(n_{2}\right)}}{\left(b_{1}+b_{2} \lambda_{i}^{\left(n_{1}\right)}\right)\left(c_{1}+c_{2} \lambda_{j}^{\left(n_{2}\right)}\right)} .
$$

We ran some experiments in the square case $n_{1}=n_{2}=\sqrt{n}$. It can be shown that about $10 n$ flops are required to solve a system of the form $M z=r$ assuming that the $L D L^{T}$ factorizations of $B$ and $C$ are available. By way of comparison, about $9 n$ flops are involved when an incomplete Cholesky (IC) preconditioner is used. In the following table we compare these two preconditioners:

\begin{tabular}{|c|c|c|}
\hline$\sqrt{n}$ & $\begin{array}{c}\text { IC } \\
\text { Iterations }\end{array}$ & $\begin{array}{c}\text { Kronecker } \\
\text { Iterations }\end{array}$ \\
\hline 16 & 14 & 19 \\
32 & 23 & 33 \\
64 & 39 & 56 \\
128 & 51 & 74 \\
256 & 66 & 93 \\
\hline
\end{tabular}

Random right hand sides were used with termination criteria $r^{T} A r \leq 10^{-6}$ where $r=b-A x$ is the residual of the approximate solution. We have no "proof" why reasonable convergence occurs before $\sqrt{n}$ steps. A plot of the spectrum of $M^{-1} A$ using (6.3) reveals that many eigenvalues of $M^{-1} A$ are clustered about 1 :

However, the clustering is not definitive enough to suggest that $O(\sqrt{n})$ convergence is provable.

The Kronecker preconditioner applied to the above model problem compares favorably with many of the other block preconditioners that are reported in Concus, Golub, and Meurant (1985). In a distributed memory environment, we suspect that the Kronecker approach may be very attractive because the preconditioner equation $C Z B^{T}=R$ is structured perfectly for parallel computation-but that is the subject of ongoing research. 


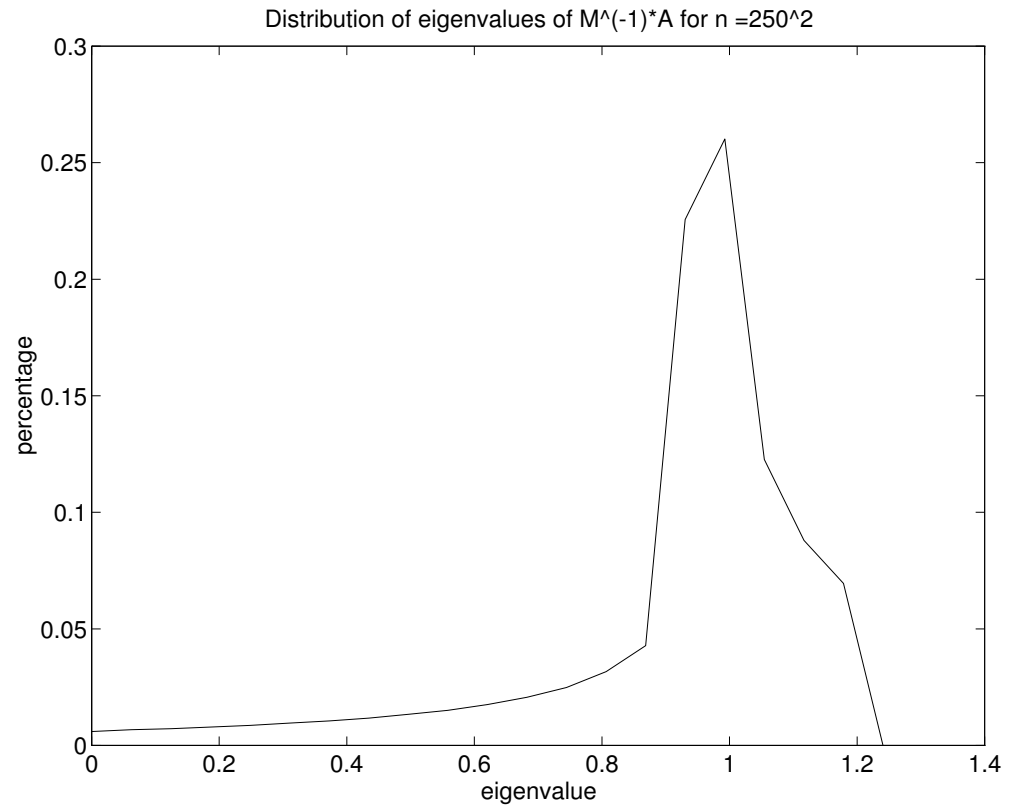

Figure 1: Distribution of Eigenvalues

\section{Acknowledgements}

Nick Trefethen of Cornell University suggested the connection between Kronecker and rank-1 approximation. Roger Horn of the University of Utah enhanced our understanding of the rearrangement operator and pointed the way to the proof of Theorem 5.6.

This research was partially supported by the Cornell Theory Center, which receives major funding from the National Science Foundation and IBM Corporation, with additional support from New York State and members of its Corporate Research Institute.

\section{References}

H.C. Andrews and J. Kane (1970). "Kronecker Matrices, Computer Implementation, and Generalized Spectra," J. Assoc. Comput. Mach. 17, 260-268.

R.H. Barham and W. Drane (1972). "An Algorithm for Least Squares Estimation of Nonlinear Parameters when Some of the Parameters are Linear," Technometrics, $14,757-766$.

R.H. Bartels and G.W. Stewart (1972). "Solution of the Equation $A X+X B=C$," Comm. ACM 15,820-826.

J.W. Brewer (1978). "Kronecker Products and Matrix Calculus in System Theory," IEEE Trans. on Circuits and Systems, 25, 772-781.

T.F. Chan (1988). "An Optimal Circulant Preconditioner for Toeplitz Systems," SIAM J. Sci. Stat. Comp., 9, 766-771.

R. Chan and X-Q Jin (1992). "A Family of Block Preconditioners for Block Systems," SIAM J. Sci. and Stat. Comp., 13, 1218-1235. 
P. Concus, G.H. Golub, and G. Meurant (1985). "Block Preconditioning for the Conjugate Gradient Method," SIAM J. Sci. Stat. Comp., 6, 220-252.

J. Cullum and R.A. Willoughby (1985). Lanczos Algorithms for Large Sparse Symmetric Eigenvalue Computations, Volume I (Theory) and II (Programs), Birkhauser, Boston.

C. de Boor (1979). "Efficient Computer Manipulation of Tensor Products," $A C M$ Trans. Math. Software, 5, 173-182.

D.W. Fausett and C. Fulton (1992). "Large Least Squares Problems Involving Kronecker Products," SIAM J. Matrix Analysis, to appear.

G.H. Golub (1973). "Some Modified Eigenvalue Problems," SIAM Review, 15, 318344.

G.H. Golub, F. Luk, and M. Overton (1981). "A Block Lanzcos Method for Computing the Singular Values and Corresponding Singular Vectors of a Matrix," ACM Trans. Math. Soft., 7, 149-169.

G.H. Golub, S. Nash, and C. Van Loan (1979). "A Hessenberg-Schur Method for the Matrix Problem $A X+X B=C$,"IEEE Trans. Auto. Cont., AC-24, 909-913.

G.H. Golub and V. Pereya (1973). "The Differentiation of PseudoInverses and Nonlinear least Squares Problems Whose Variables Separate," SIAM J. Numer. Analysis, $10,413-432$.

G.H. Golub and C. Van Loan (1989). Matrix Computations, 2nd Ed., Johns Hopkins University Press, Baltimore, MD.

A. Graham (1981). Kronecker Products and Matrix Calculus with Applications, Ellis Horwood Ltd., Chichester, England.

S.R Heap and D.J. Lindler (1986). "Block Iterative Restoration of Astronomical Images with the Massively Parallel Processor," Proc. of the First Aerospace Symposium on Massively Parallel Scientific Computation, 99-109.

H.V. Henderson, F. Pukelsheim, and S.R. Searle (1983). "On the History of the Kronecker Product," Linear and Multilinear Algebra 14, 113-120.

H.V. Henderson and S.R. Searle (1981). "The Vec-Permutation Matrix, The Vec Operator and Kronecker Products: A Review," Linear and Multilinear Algebra 9, $271-288$.

R.A. Horn and C.A. Johnson (1985). Matrix Analysis, Cambridge University Press, New York.

R.A. Horn and C.A. Johnson (1991). Topics in Matrix Analysis, Cambridge University Press, New York.

C-H Huang, J.R. Johnson, and R.W. Johnson (1991). "Multilinear Algebra and Parallel Programming," J. Supercomputing, 5, 189-217.

J. Johnson, R.W. Johnson, D. Rodriguez, and R. Tolimieri (1990). "A Methodology for Designing, Modifying, and Implementing Fourier Transform Algorithms on Various Architectures," Circuits, Systems, and Signal Processing 9, 449-500.

L. Kaufman (1975). "A Variable Projection Method for Solving Separable Nonlinear Least Squares Problems," BIT 15, 49-57.

B. Klaus and P. Horn (1990). Robot Vision. MIT Press, Cambridge, Mass.

V. Pereyra and G. Scherer (1973). "Efficient Computer Manipulation of Tensor Products with Applications to Multidimensional Approximation," Mathematics of Computation, 27, 595-604.

U.A. Rauhala (1980). "Introduction to Array Algebra," Photogrammetric Engineering and Remote Sensing, 46(2), 177-182.

P.A. Regalia and S. Mitra (1989). "Kronecker Products, Unitary Matrices, and Signal Processing Applications," SIAM Rev. 31, 586-613.

A. Swami and J. Mendel (1990). "Time and Lag Recursive Computation of Cumulants from a State-Space Model", IEEE Trans. Auto. Cont., 35, 4-17.

C. Van Loan (1992). Computational Frameworks for the Fast Fourier Transform, SIAM Publications, Philadelphia, PA. 
J.H. Wilkinson (1965). The Algebraic Eigenvalue Problem. Oxford University Press, New York. 